\title{
A Confidence-based Dominance Operator in Evolutionary Algorithms for Noisy Multiobjective Optimization Problems
}

\author{
Pruet Boonma and Junichi Suzuki \\ University of Massachusetts Boston \\ Department of Computer Science \\ 100 Morrissey Blvd. Boston, MA 02125-3393, USA \\ \{pruet, jxs\}@cs.umb.edu
}

\begin{abstract}
This paper describes a noise-aware dominance operator for evolutionary algorithms to solve the multiobjective optimization problems (MOPs) that contain noise in their objective functions. This operator takes objective value samples of given two individuals (or solution candidates), estimates the impacts of noise on the samples and determines whether it is confident enough to judge which one is superior/inferior between the two individuals. Since the proposed operator assumes no noise distributions a priori, it is well applicable to various MOPs whose objective functions follow unknown noise distributions. Experimental results show that it operates reliably in noisy MOPs and outperforms existing noise-aware dominance operators.
\end{abstract}

\section{Introduction}

This paper proposes and evaluates a noise-aware operator for evolutionary multiobjective optimization algorithms (EMOAs). An EMOA uses a population of individuals, each of which represents a solution candidate. It evolves individuals through generations and seeks the optimal solution(s) in a multiobjective optimization problem (MOP), which is formalized as follows.

$$
\left.\begin{array}{r}
\min F(x)=\left[f_{1}(\vec{x}), f_{2}(\vec{x}), \cdots, f_{m}(\vec{x})\right]^{T} \in \mathcal{O} \\
\text { subject to } \vec{x}=\left[x_{1}, x_{2}, \cdots, x_{n}\right]^{T} \in \mathcal{S}
\end{array}\right\}
$$

$\mathcal{S}$ denotes a decision variable space. $\vec{x}$ denotes a decision variable vector (or solution candidate) with respect to $\mathcal{S}$. It is called an individual in EMOAs. A function vector, $F$ : $\mathbb{R}^{n} \rightarrow \mathbb{R}^{m}$, consists of $m$ real-value objective functions, each of which produces an objective value with respect to an objective space, $\mathcal{O}$. An MOP is to find an individual(s) that minimizes objective values with subject to $\mathcal{O}$.

In an MOP, objective functions conflict with each other; there exist rarely a single individual that is optimum with respect to all objectives. Therefore, an MOP often aims to find the optimal trade-off solutions, or Pareto-optimal solutions, by seeking non-dominated individuals. An individual $\vec{x} \in \mathcal{S}$ is said to dominate an individual $\vec{y} \in \mathcal{S}$ (denoted by $\vec{x} \succ \vec{y})$ if and only if $f_{i}(\vec{x}) \leq f_{i}(\vec{y}) \forall i=1, \cdots, m$ and $f_{i}(\vec{x})<f_{i}(\vec{y}) \exists i=1, \cdots, m[11]$.

In real-world MOPs, objective functions tend to contain noise $[2,13]$. Thus, objective functions can yield different objective values from the same individual from time to time. For considering this noise, Equation 1 is revised as follows.

$$
\left.\begin{array}{rl}
\min F(x)= & {\left[f_{1}(\vec{x})+\epsilon_{1}, \cdots, f_{m}(\vec{x})+\epsilon_{m}\right]^{T} \in \mathcal{O}} \\
& \text { subject to } \vec{x}=\left[x_{1}, x_{2}, \cdots, x_{n}\right]^{T} \in \mathcal{S}
\end{array}\right\}
$$

$\epsilon_{m}$ represents noise in the $m$-th objective function. Noise in objective functions can interfere with a dominance operator, which determines dominance relationships among individuals. For example, a dominance operator may mistakenly judge that an inferior individual dominates an superior one. Defects in a dominance operator significantly degrades the performance to solve MOPs $[2,13]$.

In order to address this issue, this paper studies a new noise-aware dominance operator, called the $\alpha$-dominance operator, for EMOAs. It takes objective value samples of given two individuals, estimates the impacts of noise on the samples and determines whether it is confident enough to judge a dominance relationship between the two individuals. Unlike existing noise-aware dominance operators, the $\alpha$-dominance operator assume no noise distributions a priori. Thus, it is well applicable to a variety of MOPs whose objective functions follow unknown noise distributions.

For evaluating the $\alpha$-dominance operator, it is integrated with NSGA-II [3], a well-known EMOA, and compared with existing noise-aware dominance operators. Experimental results show that it reliably performs dominance operation in noisy MOPs and outperforms existing operators in terms of the convergence and diversity of individuals. 


\section{Background: EMOAs}

EMOAs are designed to evolve individuals toward Pareto-optima through generations with genetic operations such as crossover, mutation and selection. As described in Section 1, each individual represents a particular vector of decision values. Listing 1 shows how NSGA-II works.

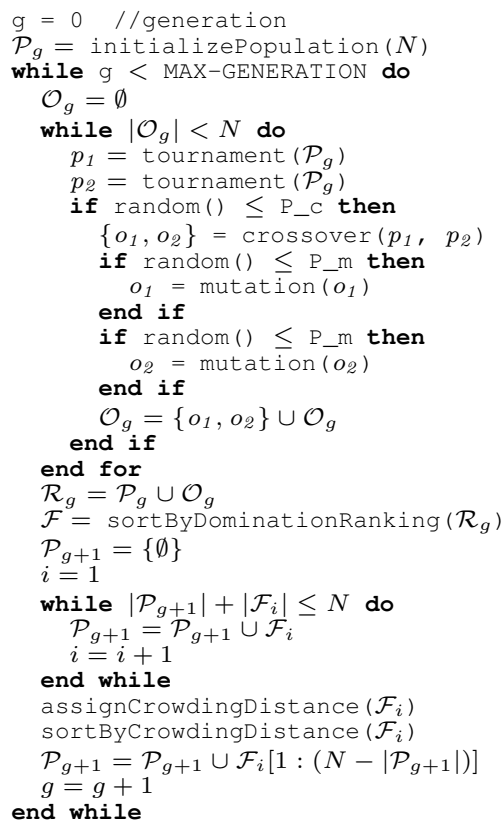

\section{Listing 1: Pseudocode of NSGA-II}

The initial population $\left(\mathcal{P}_{0}\right)$ consists of randomlygenerated $N$ individuals (Line 2 ). In each generation $(g)$, a pair of individuals, called parents $\left(p_{1}\right.$ and $\left.p_{2}\right)$, are chosen from the current population $\mathcal{P}_{g}$ using a binary tournament (Lines 6 and 7). A binary tournament randomly takes two individuals from $\mathcal{P}_{g}$, compares them based on the dominance relationship between them, and chooses a non-dominated (i.e., better) one as a parent. With the crossover rate $P_{c}$, two parents reproduce two offspring with a crossover operator (Lines 8 and 9). Each offspring performs mutation with the mutation rate $P_{m}$ (Lines 10 to 15). The binary tournament, crossover and mutation operations are performed repeatedly on $\mathcal{P}_{g}$ to produce $N$ offspring. The offspring $\left(\mathcal{O}_{g}\right)$ are combined with the parent population $\mathcal{P}_{g}$ to form $\mathcal{R}_{g}$ (Line 19).

In the selection process, $N$ individuals are selected from $2 N$ individuals in $\mathcal{R}_{g}$ as the next generation's population $\left(\mathcal{P}_{g+1}\right)$. First, the individuals in $\mathcal{R}_{g}$ are ranked based on their dominance relationships. Non-dominated individuals are on the first rank. The $i$-th rank consists of the individuals dominated only by the individuals on the $i-1$ th rank. Ranked individuals are stored in $\mathcal{F}$ (Line 20). $\mathcal{F}_{i}$ contains the $i$-th rank individuals. Then, the individuals in $\mathcal{F}$ move to $\mathcal{P}_{g+1}$ rank by rank, starting with $\mathcal{F}_{1}$ (Lines 23 to 26). If the number of individuals in $\mathcal{P}_{g+1} \cup \mathcal{F}_{i}$ is less than $N, \mathcal{F}_{i}$ moves to $\mathcal{P}_{g+1}$. Otherwise, a subset of $\mathcal{F}_{i}$ moves to $\mathcal{P}_{g+1}$. The subset is selected based on the crowding distance metric, which measures the distribution (or diversity) of individuals in the objective space (Lines 27 to 29). The metric computes the distance between two closest neighbors of an individual in each objective and sums up the distances associated with all objectives. In Line 28, the individuals in $\mathcal{F}_{i}$ are sorted based on this metric, from the ones with higher crowding distance to the ones with lower crowding distance. The individuals with higher crowding distance have higher chances to be selected to $\mathcal{P}_{g+1}$ (Line 29).

\section{Related Work}

Several existing dominance operators consider noise in objective functions [8, 10]; however, all of them assume particular noise distributions. For example, [1, 7,9] assume Gaussian noise, which follow normal distributions. [12] assumes random noise, which follow continuous uniform distributions. [5, 15] assume Poisson noise, which follow Poisson distributions. Given a noise distribution, each of existing noise-aware dominance operators statistically estimates each individual's objective value by collecting its samples. In contrast, the $\alpha$-dominance operator assumes no noise distributions a priori because, in general, it is hard to predict and model them in most (particularly, real-world) MOPs. Instead of estimating each individual's objective values, the $\alpha$-dominance operator measures the effect of noise on objective value samples and determines whether it is confident enough to compare individuals.

\section{The $\alpha$-Dominance Operator}

This section describes how the $\alpha$-dominance operator is designed (Sections 4.1 and 4.2) and how it can be integrated with existing EMOAs (Section 4.3).

\section{1. $\alpha$-Dominance Relationship}

The $\alpha$-dominance operator determines the $\alpha$-dominance relationship between given two individuals by statistically processing their objective value samples. With this operator, individual $A$ is said to $\alpha$-dominate individual $B$ (denoted by $A \succ_{\alpha} B$ ), iif:

- $A$ 's and $B$ 's objective value samples are classifiable with the statistical confidence level of $\alpha$, and

- $\mathcal{C}(A, B)=1 \wedge \mathcal{C}(B, A)<1$.

In order to examine the first condition, the $\alpha$-dominance operator classifies $A$ 's and $B$ 's objective value samples with Support Vector Machine (SVM), and measures classification error. (See Step 1 in an example shown in Figure 1.) The error $(e)$ is calculated as the ratio of the number of missclassified samples to the total number of samples. For evaluating confidence level $(\alpha)$ in a classification error, the $\alpha$ - 
dominance operator computes the classification error's confidence interval $\left(e_{\text {int }}\right)$ :

$$
e_{i n t}=e \pm t_{\alpha, n-1} \sigma
$$

$t_{\alpha, n-1}$ denotes a single-tail $t$-distribution with $\alpha$ confidence level and $n-1$ degrees of freedom. $n$ denotes the total number of samples. $\sigma$ is the standard deviation of $e$. It is approximated as follows.

$$
\sigma \cong \sqrt{\frac{e}{n}}
$$

If $e_{i n t}$ is significant (i.e., if $e_{i n t}$ does not span zero), the $\alpha$-dominance operator cannot classify $A$ 's and $B$ 's samples with the confidence level of $\alpha$. Thus, the operator determines that $\mathrm{A}$ and $\mathrm{B}$ do not $\alpha$-dominate each other. (See Step 2 in Figure 1.)

If $e_{i n t}$ is not significant (i.e., if $e_{i n t}$ spans zero), the $\alpha$ dominance operator can classify $A$ 's and $B$ 's samples with the confidence level of $\alpha$. Thus, the operator examine the second condition to determine an $\alpha$-dominance relationship (see above). It measures $\mathcal{C}$-metric [17] with a classical notion of dominance described in Section 1. $\mathcal{C}(A, B)$ denotes the fraction of individual $B$ 's samples that at least one sample of individual $A$ dominates:

$$
\mathcal{C}(A, B)=\frac{|\{b \in B \mid \exists a \in A: a \succ b\}|}{|B|}
$$

If $\mathcal{C}(A, B)=1$, all of $B$ 's samples are dominated by at least one sample of $A$. The $\alpha$-dominance operator determines $A \succ_{\alpha} B$ if $\mathcal{C}(A, B)=1$ and $\mathcal{C}(B, A)<1$. If $\mathcal{C}(A, B)<1$ and $\mathcal{C}(B, A)<1$, the operator determines neither $A \succ_{\alpha} B$ nor $B \succ_{\alpha} A$. See Step 2 in Figure 1.

Figure 1 shows an example to determine the $\alpha$ dominance relationship between two individuals, $A$ and $B$, with two objectives, $f_{1}$ and $f_{2}$, to be minimized. Individual $A$ and $B$ have seven samples each. The first step is to classify these 14 samples with SVM and compute $e_{i n t}$. SVM provides a classification vector in the objective space as a boundary to classify samples. In Figure 1, two samples of $B$ are miss-classified; $e=\frac{2}{14}$ (0.143). Thus, $\sigma \cong \sqrt{\frac{0.143}{14}}=0.1$. Assuming the confidence level $(\alpha)$ of $95 \%, e_{i n t}=0.143 \pm 1.771 * 0.1=0.143 \pm 0.1771$. Since $e_{i n t}$ spans zero, the $\alpha$-dominance operator can classify $A$ 's and $B$ 's samples with the confidence level of $95 \%$. The second step is to measure $\mathcal{C}(A, B)$ and $\mathcal{C}(B, A)$. In Figure 1, $\mathcal{C}(A, B)=1$ and $\mathcal{C}(B, A)=2 / 14<1$. Therefore, the $\alpha$-dominance operator determines $A \succ_{\alpha} B$.

\subsection{Dynamic Adjustment of Confidence Level}

The $\alpha$-dominance operator dynamically adjusts its confidence level $(\alpha)$ depending on how close individuals have converged to the Pareto-optimal front. The convergence of individuals is evaluated based on their disorderliness in the objective space. When individuals are disordered in

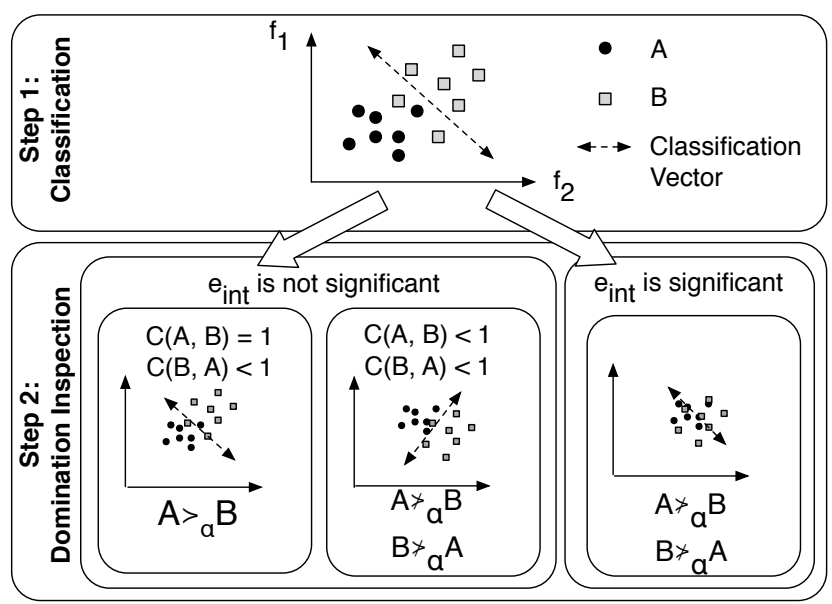

Figure 1: An Example $\alpha$-Dominance Operation

the objective space, it indicates that they have not converged enough to the Pareto-optimal front. Therefore, the $\alpha$-dominance operator maintains a low confidence level to perform dominance operation in a less strict manner and have diverse individuals explore the decision space and seek the Pareto front. Conversely, when individuals are ordered in the objective space, which indicates that individuals have converged close to the Pareto front, the $\alpha$-dominance operator increases its confidence level to perform dominance operation in a more strict manner.

The $\alpha$-dominance operator measures the disorderliness of individuals as their entropy in the objective space. To this end, a hypercube is created in the objective space. Its size is bounded by the maximum and minimum objective values yielded by individuals. The hypercube is divided to cubes. For example, Figure 2 shows that six individuals are plotted in an hypercube on a three dimensional objective space that contains eight cubes.

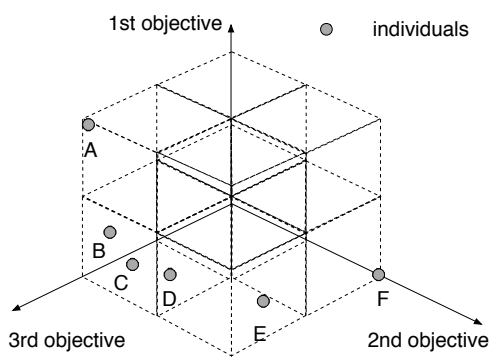

Figure 2: An Example Hypercube

Entropy of individuals $(H)$ is measured as:

$$
\begin{gathered}
H=-\sum_{i \in C} P(i) \log _{2}(P(i)) . \\
P(i)=\frac{n_{i}}{\sum_{i \in C} n_{i}}
\end{gathered}
$$


$C$ denotes a set of cubes in a hypercube, and $P(i)$ denotes the probability that individuals exist in a cube $i . n_{i}$ denotes the number of individuals in a cube $i$. Once entropy $(H)$ is obtained, it is normalized as follows:

$$
H_{o}=\frac{H}{H_{\max }}=\frac{H}{\log _{2} n}
$$

$\mathrm{n}$ denotes the total number of cubes in a hypercube. Given normalized entropy $\left(H_{o}\right)$, confidence level $(\alpha)$ is adjusted as follows:

$$
\alpha=\left(\left(\alpha_{\max }-\alpha_{\min }\right) \sqrt{1-\left(1-H_{o}\right)^{2}}\right)+\alpha_{\min }
$$

$\alpha_{\max }$ and $\alpha_{\min }$ denote the maximum and minimum confidence level, respectively. Confidence level is adjusted in a non-linear manner; a unit circle function is used to map normalized entropy to confidence level.

\subsection{Integration with Existing EMOAs}

Listing 2 shows how the $\alpha$-dominance operator works in detail.

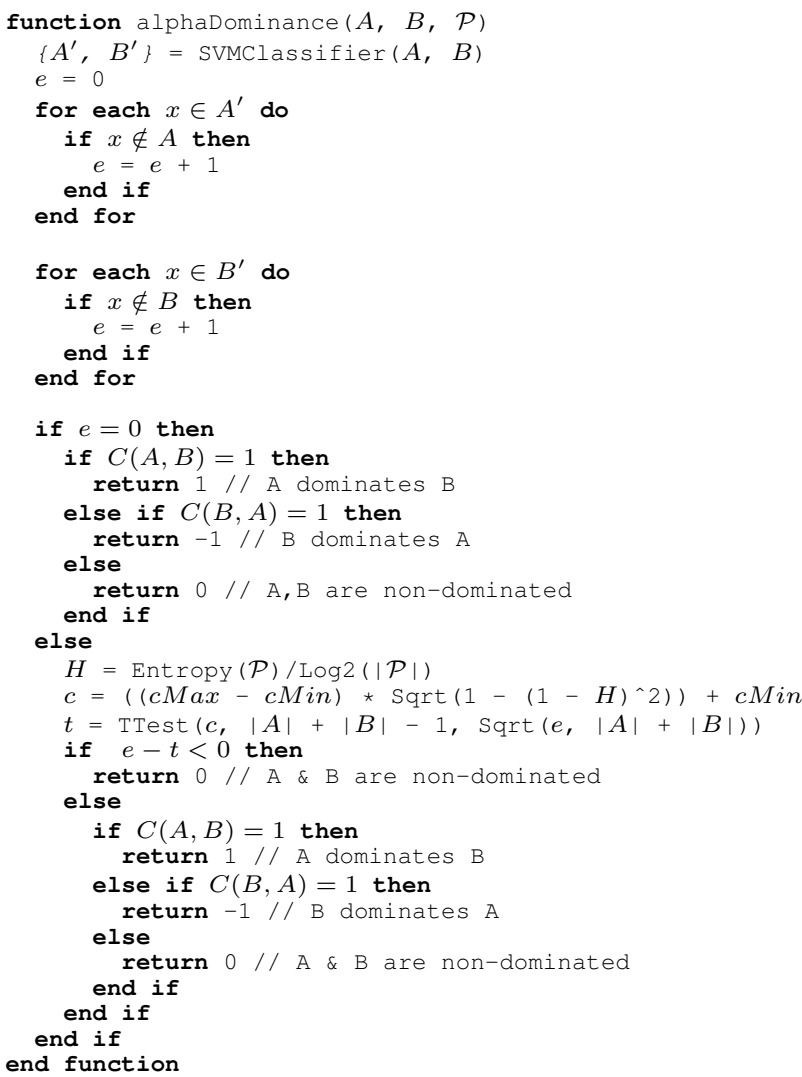

Listing 2: Pseudocode of the $\alpha$-Dominance Operator

$A$ and $B$ contain samples of individual $A$ and $B$, respectively. $\mathcal{P}$ is the current population. In Line 2 , a SVM classifies $A$ and $B$ and produces $A^{\prime}$ and $B^{\prime}$ as a result of classification. Then, classification error $(e)$ is calculated from Line 4 to 14 . Confidence level is adjusted from Line 25 to 26.
TTest ( ) performs a T-test to determine whether classification error is significant or not (Line 27).

Listing 3 shows how the default binary tournament operator (tournament ( ) ; Lines 6 and 7 in Listing 1) is modified with the $\alpha$-dominance operator.

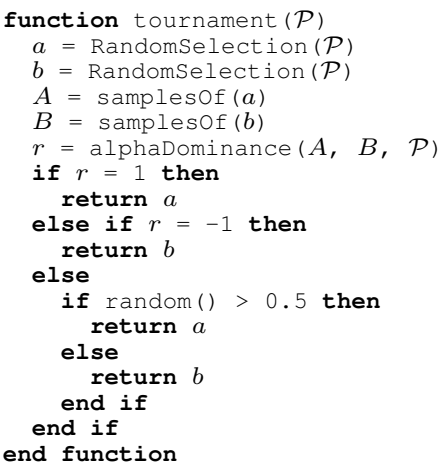

Listing 3: Pseudocode of a Binary-Tournament Operator Modified with the $\alpha$-Dominance Operator

Two individuals ( $a$ and $b$ ) are randomly taken from the current population $\mathcal{P}$ (Lines 2 and 3). Then, in Lines 4 and 5, their samples are obtained to perform $\alpha$-dominance operation at Line 6 . Based on the operation's result, one of two individuals ( $a$ or $b$ ) is returned (Line 9 to 15).

Listing 4 shows how the default ranking operator (sortByDominationRanking(); Line 20 in Listing 1) is modified with the $\alpha$-dominance operator. The modified sortByDominatinoRanking() uses findNonDominatedFront(), which finds nondominated individuals in a given population using the $\alpha$-dominance operator (Lines 11 to 17 ).

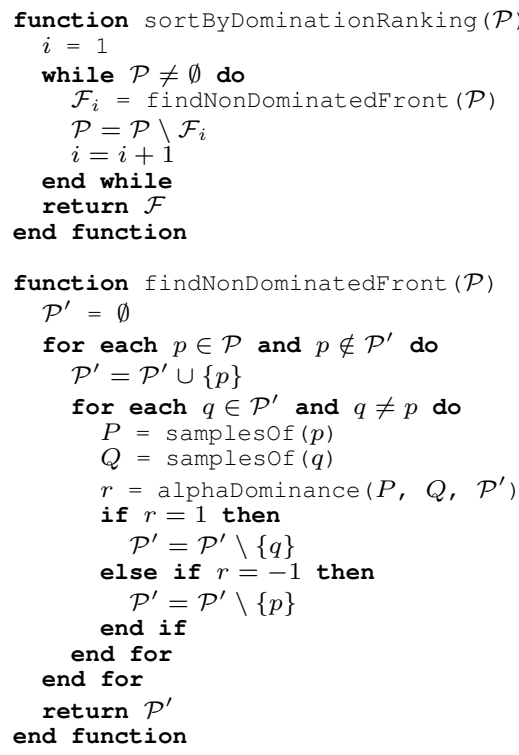

Listing 4: Pseudocode of a Ranking Operator Modified with the $\alpha$-Dominance Operator 


\begin{tabular}{|l|l|}
\hline Name & Value \\
\hline \hline Population Size & 100 \\
Number of Samples & 30 \\
Max Evaluations & 25,000 \\
Crossover Rate & 0.9 \\
Crossover Operator & $\mathrm{SBX}$ \\
Crossover Distribution Index & 20 \\
Mutation Rate & $1 /(\#$ of decision variables of an individual) \\
Mutation Operator & Polynomial mutation \\
Distribution Index for Mutation & 20 \\
Normal Dist.'s variance $\left(\sigma^{2}\right)$ & 1,4 \\
Uniform Dist.'s variance $\left(\sigma^{2}\right)$ & 1,4 \\
Poisson Dist.'s variance $(\lambda)$ & 1,4 \\
SVM Type & C-Support Vector Classification \\
SVM Kernel & Linear \\
Stopping Criteria for SVM & $1 e^{-3}$ \\
C Parameter for SVM & 1 \\
$\alpha$ min and $\alpha$ max (Equation 9) & 0.90 and 0.99 \\
\hline
\end{tabular}

Table 1: Default Parameters for EMOAs

\section{Experimental Evaluation}

In this evaluation, $\alpha$-dominance operator is compared with two noise-aware dominance operators. The evaluation was performed in jMetal [6] on a $2 \mathrm{GHz} 64$ bit dual-core processor with 2GB RAM and 64 bit JDK version 1.6.0 . $\alpha$ dominance was compared with two noise-aware dominance operator, proposed in [12] and [7] which assume continuous uniform noise and normal distribution noise, respectively which assume continuous uniform distribution noise and normal distribution noise, respectively.

In this evaluation, both noise-aware dominance operators are implemented and replace the dominance operator in NSGA-II. The variance of NSGA-II with noise-aware dominance operators which assumes uniform distribution is called NSGA-II-U while the one with noise-aware dominance operators which assumes normal distribution is called NSGA-II-N. Furthermore, the NSGA-II with $\alpha$-dominance operator is called NSGA-II-A. Following table shows default parameter of all algorithm:

\subsection{Test Problems}

In this evaluation, six well-known multiobjecitve optimization test problems are used; three from ZDT [16] family and another three from DTLZ family [4]. All three problems from ZDT family, i.e., ZDT1, ZDT2 and ZDT3, are bi-objectives problems wth 30 decision variables while the three problems from DTLZ family, i.e., DTLZ1, DTLZ3 and DTLZ7, are tri-objectives problems with 12 decision variables. ZDT1 has convex Pareto-optimal front while DTZ1 has linear Pareto-optimal front. ZDT2 and DTLZ3 have concave Pareto-optimal front. Finally, ZDT3 and DTLZ7 have discrete convex Pareto-optimal front.

\subsection{Metrics}

The proposed and comparing operators are evaluated against five metrics, namely, GD [14], IGD [14], EPSILON [18], H-CUBE and $\mathcal{C}$-Metric [17]. The first three

\begin{tabular}{|l|l|l|l|}
\hline Algorithm & Mean (msec.) & Standard Deviation & $t_{0.01,39}$ \\
\hline NSGA-II-A & 0.3166 & 0.0133 & - \\
NSGA-II-U & 0.5349 & 0.0144 & Y \\
NSGA-II-N & 0.5276 & 0.0111 & Y \\
\hline
\end{tabular}

Table 2: Comparison of Execution Time

metrics evaluates convergence, i.e., how close to the Paretooptimal front, of non-dominated front from a particular algorithm . H-CUBE evaluates the distribution of nondominated front. The last metric compare the quality of non-dominated fronts from two algorithms.

Hypercube Distribution Metric (H-CUBE) measures the distribution of non-dominated individuals by projecting each non-dominated individuals in a hypercube in the objective space (See Figure 2 for an example of hypercube. Then, the Entropy of the hypercube is measured using Equation 8. If the current set of non-dominated individuals is very diverse, e.g., only an individual located in each cube, the Entropy of the hypercube will be very high.

\subsection{Computational Cost Result}

To compare the actual execution time of each operator, NSGA-II-A, NSGA-II-N and NSGA-II-U were evaluate against DTLZ-3 problem using default parameters in Table 1; then, the time to perform 100 objective function evaluations were measure and the average of an function evaluation is calculated. 20 simulation runs were performed for each algorithm and the results are presented below:

In the table, the right most column represents the onetail significant level from $t$-distribution on 39 degree of freedom and $99 \%$ confidence level between NSGA-II-A and the other two algorithms. The simulation result shows that NSGA-II-A with $\alpha$-dominance is faster than the other two noise-aware dominance operators with $99 \%$ confidence level.

\subsection{Empirical Results with Bi-objectives Problems}

This section presents empirical results with ZDT1, ZDT2 and ZDT3 problems. In each problem, three noise distributions (Normal, Uniform and Possion distribution) and 2 noise levels (Variance of 1 and 4), measured as the variance of the noise, were evaluated on each algorithm. The results from each algorithm are presented as an average value and standard deviation as the subscription and the result in bold indicates the best result among the three algorithms. For each row in the table, one-tail significant level from $t$-distribution on 39 degree of freedom $(p-1)$ and 95\% confidence level $(\alpha)$ between the best algorithm and the other two algorithms is presented at the last column masked with *, Y indicates that the best algorithm outperforms the other two algorithm significantly. $T$

Table 3 shows result from GD metric on ZDT1, ZDT2 and ZDT3 problems. It is clear that NSGA-II-A can out performs the other two algorithms on all problem significantly. Table 4 shows result from IGD metric on the same 


\begin{tabular}{|c|c|c|c|c|}
\hline Problem & NSGA-II-N & NSGA-II-U & NSGA-II-A & * \\
\hline ZDT1-1-N & $5.78 e-011.3 e-01$ & $2.75 e-01_{2} .3 e-02$ & $1.11 \mathrm{e}-01_{2} .3 \mathrm{e}-02$ & $\mathrm{Y}$ \\
\hline ZDT1-4-N & $7.32 e-01_{2.5 e-01}$ & $2.63 e-01_{1.9 e-02}$ & $2.04 e-015.8 e-02$ & $\mathrm{Y}$ \\
\hline ZDT2-1-N & $1.20 e+005.0 e-01$ & $3.90 e-01_{1} .4 .0 e-02$ & $2.02 e-013.5 e-02$ & $\mathrm{Y}$ \\
\hline ZDT2-4-N & $1.0 .11 e+00_{2} .9 e-01$ & $3.89 e-011.5 e-02$ & $3.37 e-015.6 e-02$ & $\mathrm{Y}$ \\
\hline ZDT3-1-N & $3.60 e-018.6 e-02$ & $1.54 .0 e-01_{8.3 e-03}$ & $6.75 e-021.7 e-02$ & Y \\
\hline ZDT3-4-N & $5.21 .0 e-01_{1.6 e-01}$ & $1.57 e-01_{9.9 e-03}$ & $1.35 e-012.5 e-02$ & $\mathrm{Y}$ \\
\hline ZDT1-1-U & $5.23 e-011.4 .0 e-01$ & $3.32 e-01_{2.0 e-02}$ & $1.28 \mathrm{e}-01_{1.1 e-02}$ & $\mathrm{Y}$ \\
\hline ZDT1-4-U & $5.52 e-011.5 e-01$ & $3.32 e-01_{1.3 e-02}$ & $1.27 \mathrm{e}-01_{1} .3 \mathrm{e}-02$ & $\mathrm{Y}$ \\
\hline ZDT2-1-U & $1.09 e+003.1 e-01$ & $4.0 .43 e-01_{1} .6 e-02$ & $1.63 e-011.6 e-02$ & $\mathrm{Y}$ \\
\hline ZDT2-4-U & $1.0 .08 e+00_{3.4 e-01}$ & $4.40 e-01_{1} .2 e-02$ & $1.69 \mathrm{e}-01_{1.4 \mathrm{e}-02}$ & $\mathrm{Y}$ \\
\hline ZDT3-1-U & $3.08 e-01_{6.4 .0 e-02}$ & $2.02 e-017.3 e-03$ & $1.07 e-011.3 e-02$ & $\mathrm{Y}$ \\
\hline ZDT3-4-U & $3.20 e-01.05 .7 e-02$ & $2.03 e-01_{7} .6 e-03$ & $1.04 e-011.0 e-02$ & $\mathrm{Y}$ \\
\hline ZDT1-1-P & $7.90 e-01_{1.4 .0 e-01}$ & $3.93 e-011.7 e-02$ & $2.45 e-012.3 e-02$ & $\mathrm{Y}$ \\
\hline ZDT1-4-P & $7.70 e-01_{1.6 e-01}$ & $3.91 e-011.2 e-02$ & $2.39 e-012.5 e-02$ & $\mathrm{Y}$ \\
\hline ZDT2-1-P & $1.34 .0 e+003.4 e-01$ & $5.00 e-011.3 e-02$ & $3.11 \mathrm{e}-01_{2} .7 \mathrm{e}-02$ & $\mathrm{Y}$ \\
\hline ZDT2-4-P & $1.0 .35 e+003.1 e-01$ & $5.05 e-01_{1.3 e-02}$ & $3.14 \mathrm{e}-013.0 \mathrm{e}-02$ & $\mathrm{Y}$ \\
\hline ZDT3-1-P & $5.10 e-01_{7.8 e-02}$ & $2.54 .0 e-01_{6.1 e-03}$ & $1.86 e-01_{1.4 e-02}$ & $\mathrm{Y}$ \\
\hline ZDT3-4-P & $5.01 .0 e-019.9 e-02$ & $2.55 e-017.3 e-03$ & $1.82 \mathrm{e}-011.6 \mathrm{e}-02$ & $Y$ \\
\hline
\end{tabular}

Table 3: GD on Bi-objectives Problems

\begin{tabular}{|c|c|c|c|c|}
\hline Problem & NSGA-II-N & NSGA-II-U & NSGA-II-A & * \\
\hline ZDT1-1-N & $6.46 e-028.6 e-03$ & $6.40 e-027.5 e-03$ & $4.27 \mathrm{e}-021.0 \mathrm{e}-02$ & $\mathrm{Y}$ \\
\hline ZDT1-4-N & $4.14 \mathrm{e}-021.1 \mathrm{e}-02$ & $4.48 e-021.0 e-02$ & $5.06 e-021.8 e-02$ & $\mathrm{~N}$ \\
\hline ZDT2-1-N & $1.06 e-018.1 e-03$ & $1.05 e-017.7 e-03$ & $6.73 e-021.1 e-02$ & $\mathrm{Y}$ \\
\hline ZDT2-4-N & $8.61 e-029.0 e-03$ & $8.21 e-021.0 e-02$ & $7.94 e-022.3 e-02$ & $\mathrm{~N}$ \\
\hline ZDT3-1-N & $7.15 e-027.5 e-03$ & $7.23 e-028.3 e-03$ & $4.18 \mathrm{e}-021.0 \mathrm{e}-02$ & $\mathrm{Y}$ \\
\hline ZDT3-4-N & $5.78 e-021.2 e-02$ & $4.66 \mathrm{e}-021.2 \mathrm{e}-02$ & $6.33 e-02_{2} .0 e-02$ & $\mathrm{Y}$ \\
\hline ZDT1-1-U & $8.57 e-026.6 e-03$ & $8.58 e-026.0 e-03$ & $4.85 e-024.8 e-03$ & $\mathrm{Y}$ \\
\hline ZDT1-4-U & $8.60 e-025.5 e-03$ & $8.66 e-024.5 e-03$ & $4.68 e-024.9 e-03$ & $\mathrm{Y}$ \\
\hline ZDT2-1-U & $1.25 e-017.7 e-03$ & $1.24 e-01_{6} .9 e-03$ & $5.89 e-025.1 e-03$ & $\mathrm{Y}$ \\
\hline ZDT2-4-U & $1.26 e-015.1 e-03$ & $1.23 e-017.0 e-03$ & $6.05 e-024.5 e-03$ & $\mathrm{Y}$ \\
\hline ZDT3-1-U & $1.05 e-016.2 e-03$ & $1.07 e-017.1 e-03$ & $7.12 e-026.9 e-03$ & Y \\
\hline ZDT3-4-U & $1.06 e-01_{5} .8 e-03$ & $1.07 e-01_{6.7 e-03}$ & $7.04 e-025.1 e-03$ & Y \\
\hline ZDT1-1-P & $1.05 e-017.9 e-03$ & $1.04 e-01_{8.4 e-03}$ & $7.94 e-029.5 e-03$ & $\mathrm{Y}$ \\
\hline ZDT1-4-P & $1.05 e-01_{5.7 e-03}$ & $1.07 e-01_{5.8 e-03}$ & $7.78 e-029.3 e-03$ & $\mathrm{Y}$ \\
\hline ZDT2-1-P & $1.42 e-01_{6.0 e-03}$ & $1.42 e-01_{5.8 e-03}$ & $9.84 e-029.6 e-03$ & $\mathrm{Y}$ \\
\hline ZDT2-4-P & $1.44 e-01_{6.4 e-03}$ & $1.41 e-01_{9.4 e-03}$ & $9.94 e-029.0 e-03$ & $\mathrm{Y}$ \\
\hline ZDT3-1-P & $1.37 e-01_{6.2 e-03}$ & $1.38 e-01_{5} .5 e-03$ & $1.10 e-017.9 e-03$ & $\mathrm{Y}$ \\
\hline ZDT3-4-P & $1.39 e-016.5 e-03$ & $1.38 e-017.3 e-03$ & $1.09 e-01_{7.7 e-03}$ & $\mathrm{Y}$ \\
\hline
\end{tabular}

Table 4: IGD on Bi-objectives Problems

\begin{tabular}{|c|c|c|c|c|}
\hline Problem & NSGA-II-N & NSGA-II-U & NSGA-II-A & * \\
\hline ZDT1-1-N & $2.30 e+00_{2.8 e-01}$ & $2.29 e+00_{2.5 e-01}$ & $1.61 \mathrm{e}+00_{3.5 e-01}$ & $\mathrm{Y}$ \\
\hline ZDT1-4-N & $1.42 \mathrm{e}+003.8 \mathrm{e}-01$ & $1.57 e+003.4 e-01$ & $1.77 e+00_{5} .7 e-01$ & $\mathrm{~N}$ \\
\hline ZDT2-1-N & $3.98 e+00_{2.6 e-01}$ & $3.95 e+00_{2.5 e-01}$ & $2.72 e+003.5 e-01$ & $\mathrm{Y}$ \\
\hline ZDT2-4-N & $3.25 e+00_{2.8 e-01}$ & $3.11 e+00_{3.4 e-01}$ & $3.09 \mathrm{e}+00_{7.8 \mathrm{e}-01}$ & $\mathrm{~N}$ \\
\hline ZDT3-1-N & $2.80 e+00_{2} .3 e-01$ & $2.72 e+00_{2.9 e-01}$ & $1.73 e+00_{3.6 e-01}$ & $\mathrm{Y}$ \\
\hline ZDT3-4-N & $2.12 e+00_{3.3 e-01}$ & $1.87 e+00_{4.6 e-01}$ & $2.49 e+00_{6.1 e-01}$ & Y \\
\hline ZDT1-1-U & $2.89 e+00_{2.2 e-01}$ & $2.88 e+00_{2.1 e-01}$ & $1.74 \mathrm{e}+00_{1.9 e-01}$ & $\mathrm{Y}$ \\
\hline ZDT1-4-U & $2.89 e+0_{2} .0 e-01$ & $2.90 e+001.7 e-01$ & $1.66 e+00_{1.9 e}-01$ & Y \\
\hline ZDT2-1-U & $4.54 e+00_{2.5 e-01}$ & $4.54 e+00_{2.4 e-01}$ & $2.48 \mathrm{e}+00_{1.7 \mathrm{e}-01}$ & $\mathrm{Y}$ \\
\hline ZDT2-4-U & $4.59 e+00_{1.7 e-01}$ & $4.49 e+0_{2} .3 e-01$ & $2.53 e+00_{1.5 e-01}$ & $\mathrm{Y}$ \\
\hline ZDT3-1-U & $3.23 e+003.1 e-01$ & $3.31 e+003.2 e-01$ & $2.05 \mathrm{e}+003.2 \mathrm{e}-01$ & Y \\
\hline ZDT3-4-U & $3.26 e+00_{2.8 e-01}$ & $3.24 e+00_{2.7 e-01}$ & $2.03 e+00_{3.2 e}-01$ & $\mathrm{Y}$ \\
\hline ZDT1-1-P & $3.32 e+00_{2.8 e-01}$ & $3.31 e+0_{2} .9 e-01$ & $2.55 e+00_{3.5 e-01}$ & $\mathrm{Y}$ \\
\hline ZDT1-4-P & $3.34 e+00_{2.3 e-01}$ & $3.39 e+00_{2.0 e-01}$ & $2.50 e+00_{3.3 e-01}$ & $\mathrm{Y}$ \\
\hline ZDT2-1-P & $4.98 e+001.8 e-01$ & $4.98 e+001.9 e-01$ & $3.69 \mathrm{e}+00_{3.0 \mathrm{e}-01}$ & Y \\
\hline ZDT2-4-P & $5.04 e+00_{2.3 e-01}$ & $4.94 e+00_{3.3 e-01}$ & $3.72 \mathrm{e}+00_{2.9 e}-01$ & $\mathrm{Y}$ \\
\hline ZDT3-1-P & $3.71 e+00_{2.8 e-01}$ & $3.73 e+00_{2.6 e-01}$ & $2.86 e+003.6 e-01$ & $\mathrm{Y}$ \\
\hline ZDT3-4-P & $3.78 e+00_{2.8 e-01}$ & $3.72 e+00_{2.8 e-01}$ & $2.68 \mathrm{e}+00_{3.9 e-01}$ & $\mathrm{Y}$ \\
\hline
\end{tabular}

Table 5: EPSILON on Bi-objectives Problems

\begin{tabular}{|c|c|c|c|c|}
\hline Problem & NSGA-II-N & NSGA-II-U & NSGA-II-A & ${ }^{*}$ \\
\hline $\begin{array}{l}\text { ZDT1-1-N } \\
\end{array}$ & $2.59 e-011.9 e-02$ & $2.37 e-01_{2} .0 e-02$ & $1.93 e-011.7 e-02$ & $\mathrm{Y}$ \\
\hline ZDT1-4-N & $3.09 e-01_{6.7 e-02}$ & $3.15 e-01_{3.1 e-02}$ & $2.18 \mathrm{e}-01_{1} .8 \mathrm{e}-02$ & $\mathrm{Y}$ \\
\hline ZDT2-1-N & $3.41 e-011.9 e-01$ & $2.35 e-01_{1.8 e-02}$ & $1.96 e-011.8 e-02$ & $\mathrm{Y}$ \\
\hline ZDT2-4-N & $3.08 e-01_{6.2 e-02}$ & $2.94 e-01_{3.5 e-02}$ & $2.15 e-012.4 e-02$ & $\mathrm{Y}$ \\
\hline ZDT3-1-N & $2.66 e-01_{2} .7 e-02$ & $2.42 e-01_{1.2 e-02}$ & $1.97 e-011.5 e-02$ & $\mathrm{Y}$ \\
\hline ZDT3-4-N & $3.17 e-014.7 e-02$ & $3.25 e-01_{2.8 e-02}$ & $2.22 \mathrm{e}-012.1 \mathrm{e}-02$ & $\mathrm{Y}$ \\
\hline ZDT1-1-U & $2.51 e-011.9 e-02$ & $2.31 \mathrm{e}-011.4 \mathrm{e}-02$ & $3.08 e-017.6 e-02$ & $\mathrm{Y}$ \\
\hline ZDT1-4-U & $2.53 e-01_{2.8 e}-02$ & $2.34 \mathrm{e}-011.0 \mathrm{e}-02$ & $3.01 e-018.2 e-02$ & $\mathrm{Y}$ \\
\hline ZDT2-1-U & $3.01 e-019.6 e-02$ & $2.34 e-012.0 e-02$ & $4.37 e-01_{1.5 e-01}$ & $\mathrm{Y}$ \\
\hline ZDT2-4-U & $3.37 e-011.9 e-01$ & $2.46 e-013.0 e-02$ & $4.50 e-011.5 e-01$ & $\mathrm{Y}$ \\
\hline ZDT3-1-U & $2.63 e-013.5 e-02$ & $2.37 \mathrm{e}-01_{1.6 e-02}$ & $2.55 e-01_{5} .5 e-02$ & $\mathrm{Y}$ \\
\hline ZDT3-4-U & $2.57 e-01_{2} .8 e-02$ & $2.33 \mathrm{e}-01_{1.2 \mathrm{e}-02}$ & $2.68 e-01_{6.4 e-02}$ & $\mathrm{Y}$ \\
\hline ZDT1-1-P & $2.57 e-01_{2} .5 e-02$ & $2.45 e-01_{2} .0 e-02$ & $2.09 e-015.5 e-02$ & $\mathrm{Y}$ \\
\hline ZDT1-4-P & $2.66 e-012.7 e-02$ & $2.39 e-01_{2} .0 e-02$ & $1.96 e-012.4 e-02$ & $\mathrm{Y}$ \\
\hline ZDT2-1-P & $3.12 e-011.4 e-01$ & $2.37 e-011.8 e-02$ & $2.05 e-016.8 e-02$ & $\mathrm{Y}$ \\
\hline ZDT2-4-P & $2.97 e-016.7 e-02$ & $2.46 e-011.9 e-02$ & $2.11 \mathrm{e}-013.4 \mathrm{e}-02$ & $\mathrm{Y}$ \\
\hline ZDT3-1-P & $2.54 e-01_{1.8 e-02}$ & $2.44 e-011.3 e-02$ & $2.00 e-01_{1} .8 e-02$ & $\mathrm{Y}$ \\
\hline ZDT3-4-P & $2.57 e-01_{2} .0 e-02$ & $2.45 e-01_{1.7 e-02}$ & $2.01 \mathrm{e}-01_{1.7 \mathrm{e}-02}$ & $\mathrm{Y}$ \\
\hline
\end{tabular}

Table 6: H-CUBE on Bi-objectives Problems

\begin{tabular}{|c|c|c|c|c|}
\hline Problem & NSGA-II-N & NSGA-II-U & NSGA-II-A & $*$ \\
\hline DTLZ1-1-N & $1.33 e+025.4 e+00$ & $1.33 e+025.0 e+00$ & $7.38 \mathrm{e}+018.2 \mathrm{e}+00$ & $\mathrm{Y}$ \\
\hline DTLZ1-4-N & $1.34 e+026.7 e+00$ & $1.36 e+027.0 e+00$ & $7.31 e+017.1 e+00$ & $\mathrm{Y}$ \\
\hline DTLZ3-1-N & $1.60 e+026.1 e+00$ & $1.62 e+026.8 e+00$ & $8.02 e+01_{7.0 e+00}$ & $\mathrm{Y}$ \\
\hline DTLZ3-4-N & $1.61 e+026.3 e+00$ & $1.62 e+024.9 e+00$ & $8.39 e+01_{5} .6 e+00$ & $\mathrm{Y}$ \\
\hline DTLZ7-1-N & $3.99 e-01_{1} .0 e-01$ & $3.35 e-011.7 e-02$ & $8.86 e-021.7 e-02$ & $\mathrm{Y}$ \\
\hline DTLZ7-4-N & $5.03 e-011.5 e-01$ & $3.50 e-011.7 e-02$ & $2.17 \mathrm{e}-013.4 \mathrm{e}-02$ & $\mathrm{Y}$ \\
\hline DTLZ1-1-U & $1.34 e+025.8 e+00$ & $1.34 e+026.0 e+00$ & $7.31 e+01_{7.3 e}+00$ & Y \\
\hline DTLZ1-4-U & $1.36 e+026.3 e+00$ & $1.33 e+025.9 e+00$ & $7.44 \mathrm{e}+01_{5.2 \mathrm{e}}+00$ & $\mathrm{Y}$ \\
\hline DTLZ3-1-U & $1.64 e+027.3 e+00$ & $1.62 e+027.2 e+00$ & $8.07 e+018.5 e+00$ & $\mathrm{Y}$ \\
\hline DTLZ3-4-U & $1.62 e+026.9 e+00$ & $1.62 e+025.6 e+00$ & $8.11 \mathrm{e}+01_{7.5 e+00}$ & Y \\
\hline DTLZ7-1-U & $3.98 e-01_{1} .0 e-01$ & $3.71 e-01_{1.2 e-02}$ & $1.21 \mathrm{e}-011.2 \mathrm{e}-02$ & $\mathrm{Y}$ \\
\hline DTLZ7-4-U & $3.94 e-015.8 e-02$ & $3.71 e-011.1 e-02$ & $1.16 e-011.5 e-02$ & $\mathrm{Y}$ \\
\hline DTLZ1-1-P & $1.34 e+025.0 e+00$ & $1.35 e+027.7 e+00$ & $7.35 e+018.3 e+00$ & $\mathrm{Y}$ \\
\hline DTLZ1-4-P & $1.34 e+026.7 e+00$ & $1.36 e+026.9 e+00$ & $7.58 \mathrm{e}+01_{6.7 e}+00$ & $\mathrm{Y}$ \\
\hline DTLZ3-1-P & $1.63 e+028.0 e+00$ & $1.62 e+024.9 e+00$ & $8.36 e+01_{7.0 e+00}$ & $\mathrm{Y}$ \\
\hline DTLZ3-4-P & $1.62 e+026.2 e+00$ & $1.61 e+026.4 e+00$ & $8.26 e+017.0 e+00$ & $\mathrm{Y}$ \\
\hline DTLZ7-1-P & $4.29 e-015.0 e-02$ & $4.07 e-01_{1} .3 e-02$ & $1.97 \mathrm{e}-011.9 \mathrm{e}-02$ & $\mathrm{Y}$ \\
\hline DTLZ7-4-P & $4.54 e-01_{1.2 e-01}$ & $4.03 e-011.3 e-02$ & $2.00 e-011.7 \mathrm{e}-02$ & $\mathrm{Y}$ \\
\hline
\end{tabular}

Table 7: GD on Tri-objectives Problems

\begin{tabular}{|c|c|c|c|c|}
\hline Problem & NSGA-II-N & NSGA-II-U & NSGA-II-A & * \\
\hline DTLZ1-1-N & $8.70 e+00_{6.0 e-01}$ & $8.43 e+00_{8.2 e-01}$ & $6.72 \mathrm{e}+00_{7.8 \mathrm{e}-01}$ & \\
\hline DTLZ1-4-N & $8.71 e+007.7 e-01$ & $8.64 e+009.9 e-01$ & $6.63 e+006.8 e-01$ & Y \\
\hline DTLZ3-1-N & $1.17 e+019.4 e-01$ & $1.17 e+011.1 e+00$ & $7.22 \mathrm{e}+007.1 \mathrm{e}-01$ & Y \\
\hline DTLZ3-4-N & $1.20 e+01_{6.4 e-01}$ & $1.17 e+01_{9.7 e-01}$ & $7.60 e+00_{5.2 e}-01$ & Y \\
\hline DTLZ7-1-N & $6.36 e-024.8 e-03$ & $6.24 e-025.7 e-03$ & $2.58 \mathrm{e}-023.9 \mathrm{e}-03$ & Y \\
\hline DTLZ7-4-N & $6.19 e-025.8 e-03$ & $6.22 e-026.2 e-03$ & $4.73 e-028.3 e-03$ & Y \\
\hline DTLZ1-1-U & $8.54 e+009.9 e-01$ & $8.64 e+009.6 e-01$ & $6.64 e+00_{7.0 e}-01$ & Y \\
\hline DTLZ1-4-U & $8.66 e+001.0 e+00$ & $8.80 e+009.1 e-01$ & $6.86 e+00_{4.7 e-01}$ & Y \\
\hline DTLZ3-1-U & $1.20 e+01_{1.2 e+00}$ & $1.19 e+01_{6.7 e-01}$ & $7.33 e+00_{7.5 e-01}$ & Y \\
\hline DTLZ3-4-U & $1.18 e+019.4 e-01$ & $1.17 e+019.1 e-01$ & $7.39 e+00_{7.1 e}-01$ & Y \\
\hline DTLZ7-1-U & $7.15 e-023.8 e-03$ & $7.01 e-025.9 e-03$ & $3.31 e-022.6 e-03$ & Y \\
\hline DTLZ7-4-U & $7.05 e-025.1 e-03$ & $7.11 e-024.0 e-03$ & $3.20 e-022.8 e-03$ & Y \\
\hline DTLZ1-1-P & $8.62 e+008.5 e-01$ & $8.71 e+00_{1.0 e+00}$ & $6.61 e+00_{7.4 e-01}$ & Y \\
\hline DTLZ1-4-P & $8.69 e+008.1 e-01$ & $8.60 e+009.0 e-01$ & $6.83 e+006.5 e-01$ & $\mathrm{Y}$ \\
\hline DTLZ3-1-P & $1.19 e+01_{1.0 e+00}$ & $1.19 e+011.1 e+00$ & $7.62 e+00_{6.5 e}-01$ & Y \\
\hline DTLZ3-4-P & $1.18 e+019.4 e-01$ & $1.17 e+01_{9.2 e-01}$ & $7.42 \mathrm{e}+00_{6.8 \mathrm{e}-01}$ & $\mathrm{Y}$ \\
\hline DTLZ7-1-P & $8.20 e-023.9 e-03$ & $7.94 e-024.1 e-03$ & $4.85 e-023.9 e-03$ & $\mathrm{~V}$ \\
\hline DTLZ7-4-P & $8.22 e-023.8 e-03$ & $8.08 e-022.9 e-03$ & $4.88 \mathrm{e}-023.8 \mathrm{e}-03$ & $\mathrm{Y}$ \\
\hline
\end{tabular}

Table 8: IGD on Tri-objectives Problems

problems. Except the ZDT1-4-N, ZDT3-4-N and ZDT2-4$\mathrm{N}$ problems, NSGA-II-A outperforms the other two algorithms significantly. For ZDT1-4-N and ZDT2-4-N, the results are inconclusive while ZDT3-4-N is the only problem that NSGA-II-U can outperform NSGA-II-A significantly. Table 5 shows result from EPSILON metric on the same problem. Again, NSGA-II-A can outperform the other algorithms except on ZDT1-4-N and ZDT3-4-N. The experimental results show that $\alpha$-dominance allows EMOA to find better non-dominated individuals in term of convergence.

Table 6 shows result of Hypercube metric on ZDT1, ZDT2 and ZDT3 problems. For the problems with Uniform distribution, NSGA-II-U can outperform the other two algorithms because the dominance operator in NSGA-II-U assumes Uniform distribution noise, thus, it is not surprised that NSGA-II-U can tolerance the effect of this kind of noise better than the other algorithms. Nevertheless, on the problems with Poisson distribution noise, or even Normal distribution noise, NSGA-II-A can outperforms the others significantly. $\alpha$-domiance allows EMOA to find diverse nondominated individuals on different types of noise distribution because it does not assume any noise distribution.

Table 11 shows result of coverage metric on ZDT1, ZDT2 and ZDT3 problems. Compared NSGA-II-A with the other algorithms, individuals from NSGA-II-A dominate some of the individuals from the other algorithms and are not dominated by the individuals from the other algorithms. Only in ZDT1-4-N and ZDT3-4-N problems that some individual from NSGA-II-A are dominated by either 


\begin{tabular}{|c|c|c|c|c|}
\hline Problem & NSGA-II-N & NSGA-II-U & NSGA-II-A & * \\
\hline DTLZ1-1-N & $2.89 e+022.3 e+01$ & $2.84 e+022.8 e+01$ & $2.20 \mathrm{e}+024.2 \mathrm{e}+01$ & \\
\hline DTLZ1-4-N & $2.88 e+022.0 e+01$ & $2.90 e+022.5 e+01$ & $2.17 e+022.7 e+01$ & $\mathrm{Y}$ \\
\hline DTLZ3-1-N & $7.79 e+024.6 e+01$ & $7.88 e+025.5 e+01$ & $4.73 e+025.5 e+01$ & Y \\
\hline DTLZ3-4-N & $7.99 e+023.9 e+01$ & $7.86 e+025.5 e+01$ & $4.99 e+025.5 e+01$ & $\mathrm{Y}$ \\
\hline DTLZ7-1-N & $1.11 e+017.8 e-01$ & $1.08 e+01_{9.7 e-01}$ & $5.14 e+00_{7.0 e-01}$ & $\mathrm{Y}$ \\
\hline DTLZ7-4-N & $1.05 e+017.6 e-01$ & $1.06 e+01_{9.6 e-01}$ & $8.23 e+00_{1} .3 e+00$ & $\mathrm{Y}$ \\
\hline DTLZ1-1-U & $2.89 e+022.8 e+01$ & $2.89 e+022.8 e+01$ & $2.12 \mathrm{e}+022.9 \mathrm{e}+01$ & $\mathrm{Y}$ \\
\hline DTLZ1-4-U & $2.92 e+022.5 e+01$ & $2.91 e+022.7 e+01$ & $2.26 e+022.5 e+01$ & $\mathrm{Y}$ \\
\hline DTLZ3-1-U & $7.98 e+024.8 e+01$ & $7.96 e+025.4 e+01$ & $4.78 e+026.4 e+01$ & $\mathrm{Y}$ \\
\hline DTLZ3-4-U & $7.95 e+025.0 e+01$ & $7.74 e+023.4 e+01$ & $4.72 e+025.7 e+01$ & $\mathrm{Y}$ \\
\hline DTLZ7-1-U & $1.16 e+016.9 e-01$ & $1.13 e+011.0 e+00$ & $6.17 e+00_{5} .0 e-01$ & $\mathrm{Y}$ \\
\hline DTLZ7-4-U & $1.14 e+01_{9.3 e-01}$ & $1.15 e+017.7 e-01$ & $6.00 e+00_{6.2 e}-01$ & $\mathrm{Y}$ \\
\hline DTLZ1-1-P & $2.93 e+022.0 e+01$ & $2.93 e+022.4 e+01$ & $2.18 \mathrm{e}+024.2 \mathrm{e}+01$ & $\mathrm{Y}$ \\
\hline DTLZ1-4-P & $2.89 e+022.4 e+01$ & $2.86 e+022.9 e+01$ & $2.32 e+023.3 e+01$ & Y \\
\hline DTLZ3-1-P & $7.86 e+026.2 e+01$ & $8.04 e+025.3 e+01$ & $4.95 e+025.5 e+01$ & $\mathrm{Y}$ \\
\hline DTLZ3-4-P & $7.82 e+025.9 e+01$ & $7.85 e+025.2 e+01$ & $4.84 e+026.2 e+01$ & $\mathrm{Y}$ \\
\hline DTLZ7-1-P & $1.20 e+017.1 e-01$ & $1.17 e+01_{9.1 e-01}$ & $6.59 e+00_{7.5 e-01}$ & $\mathrm{Y}$ \\
\hline DTLZ7-4-P & $1.22 e+01_{8.5 e-01}$ & $1.19 e+01_{5.3 e-01}$ & $6.35 e+008.1 e-01$ & $\mathrm{Y}$ \\
\hline
\end{tabular}

Table 9: EPSILON on Tri-objectives Problems

\begin{tabular}{|c|c|c|c|c|}
\hline Problem & NSGA-II-N & NSGA-II-U & NSGA-II-A & \\
\hline DTLZ1-1-N & $7.56 e-01_{2} .9 e-02$ & $7.54 e-013.1 e-02$ & $7.26 e-013.5 e-02$ & $\mathrm{Y}$ \\
\hline DTLZ1-4-N & $7.56 e-013.0 e-02$ & $7.61 e-01_{4} .3 e-02$ & $7.30 \mathrm{e}-013.4 \mathrm{e}-02$ & 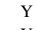 \\
\hline DTLZ3-1-N & $7.65 e-01_{4.2 e-02}$ & $7.57 e-01_{3.9 e-02}$ & $7.16 \mathrm{e}-01_{3.1 \mathrm{e}-02}$ & \\
\hline DTLZ3-4-N & $7.57 e-013.6 e-02$ & $7.61 e-01_{3.3 e-02}$ & $7.21 \mathrm{e}-01_{3.9 e-02}$ & $\mathrm{Y}$ \\
\hline DTLZ7-1-N & $1.70 e-01_{1.5 e-02}$ & $1.59 e-011.1 e-02$ & $1.56 e-01_{4.3 e-03}$ & \\
\hline DTLZ7-4-N & $1.97 e-011.6 e-02$ & $1.83 e-011.4 e-02$ & $1.62 \mathrm{e}-018.8 \mathrm{e}-03$ & $\mathrm{Y}$ \\
\hline DTLZ1-1-U & $7.69 e-013.1 e-02$ & $7.47 e-013.7 e-02$ & $7.04 \mathrm{e}-01_{1.1 \mathrm{e}-01}$ & $\mathrm{Y}$ \\
\hline DTLZ1-4-U & $7.62 e-01_{3.7 e-02}$ & $7.55 e-0$ & $7.13 e-013.7 e-02$ & 1 \\
\hline DTLZ3-1-U & $7.60 e-013.8 e-02$ & $7.56 e-01_{3.6 e-02}$ & $7.21 \mathrm{e}-01_{3.9 e-02}$ & Y \\
\hline DTLZ3-4-U & $7.59 e-01_{3.1 e-02}$ & $7.55 e-01_{3.4 e-02}$ & $7.36 e-01_{3.6 e-02}$ & $\mathrm{Y}$ \\
\hline DTLZ7-1-U & $1.59 e-01_{1} .6 e-02$ & $1.53 e-016.0 e$ & $2.30 e-01_{8.1 e-02}$ & $\mathrm{Y}$ \\
\hline DTLZ7-4-U & $1.57 e-01_{1} .3 e-02$ & $1.52 \mathrm{e}-019.3$ & $1.96 e-01_{3.2 e-02}$ & Y \\
\hline DTLZ1-1-P & $7.57 e-013.7 e-02$ & $7.71 e-01_{4.4 e-02}$ & $7.28 \mathrm{e}-01_{3.8 \mathrm{e}-02}$ & $\mathrm{Y}$ \\
\hline DTLZ1-4-P & $7.57 e-01_{2.6 e-02}$ & $7.72 e-014$ & $7.03 e-016.8 e-02$ & Y \\
\hline DTLZ3-1-P & $7.60 e-013.3 e-02$ & $7.53 e-014.2 e-02$ & $7.31 e-013.7 e-02$ & \\
\hline DTLZ3-4-P & $7.68 e-0$ & $7.58 e-$ & $7.38 e-01_{4.3 e-02}$ & Y \\
\hline DT & $1.67 e-011.4 e-02$ & $1.58 e-01$ & $1.56 \mathrm{e}-01_{4.1 \mathrm{e}-03}$ & \\
\hline DTLZ7-4-P & $1.71 e-012.1 e-02$ & $1.59 e-017.1 e-03$ & $1.56 e-016.4 e-03$ & \\
\hline
\end{tabular}

Table 10: H-CUBE on Tri-objectives Problems

NSGA-II-U's or NSGA-II-N's individuals. $\alpha$-dominance allows EMOA to find better non-dominated individuals.

\subsection{Empirical Results with Tri-objectives Problems}

This section presents empirical results with DTLZ1, DTLZ3 and DTLZ7 problems. All of the experiment setting is the same as the previous section.

Table 7, 8 and 9 show result from GD, IGD and EPSILON metrics on DTLZ1, DTLZ3 and DTLZ7 problems, respectively. It is clear that NSGA-II-A can out performs the other two algorithms on all problem significantly. The experimental results show that $\alpha$-dominance allows EMOA to find better non-dominated individuals in terms of convergence in high-dimensional problems.

Table 10 shows result of Hypercube metric on DTLZ1, DTLZ3 and DTLZ7 problems. NSGA-II-U can outperform NSGA-II-A in only two problems with Uniform distribution. In particular, the dominance operator in NSGA-II$\mathrm{U}$ assumes Uniform distribution noise, thus, it is not surprised that NSGA-II-U can tolerance the effect of this kind of noise better than the other algorithms. Nevertheless, on most of the problems with Poisson distribution noise, all of the problems with Normal distribution noise, and four problems with Uniform distribution noise, NSGA-II-A can outperforms the others significantly. $\alpha$-domiance allows EMOA to find diverse non-dominated individuals on different types of noise distributions because it does not assume any noise distribution.

Table 12 shows result of coverage metric on DTLZ1, DTLZ3 and DTLZ7 problems. Individuals from NSGAII-A can dominate individuals from the other algorithms in most cases. Moreover, individuals from NSGA-II-A are never be dominated by individuals from the other algorithms. $\alpha$-dominance allows EMOA to find better nondominated individuals.

\section{Conclusions}

This paper proposes and evaluates $\alpha$-dominance, a noiseaware dominance operator. Contrast with previous works, $\alpha$-dominance does not assume any noise distribution, instead, it uses classifier to justify whehter an individual dominate the other individual. Evaluation results show that $\alpha$ dominance allows EMOA to find better non-dominated individuals in terms of convergence and diversity.

\section{References}

[1] M. Babbar, A. Lakshmikantha, and D. E. Goldberg. A modified NSGA-II to solve noisy multiobjective problems. In Proc. of ACM Genetic and Evol. Comp. Conf., 2003.

[2] R. J. Carroll, D. Ruppert, L. A. Stefanski, and C. Crainiceanu. Measurement Error in Nonlinear Models: A Modern Perspective, Second Edition. CRC, 2006.

[3] K. Deb, S. Agrawal, A. Pratab, and T. Meyarivan. A fast elitist non-dominated sorting genetic algorithm for multiobjective optimization: NSGA-II. In Proc. of Int'l Parallel Problem Solving from Nature Conf., 2000.

[4] K. Deb, L. Thiele, M. Laumanns, and E. Zitzler. Scalable multi-objective optimization test problems. In Proc. of IEEE Congress on Evol. Comp., 2002.

[5] K. Delibrasis, P. Undrill, and G. Cameron. Genetic algorithm implementation of stack filter design for image restoration. In Proc. of Vision, image and signal proc., 1996.

[6] J. J. Durillo, A. J. Nebro, F. Luna, B. Dorronsoro, and E. Alba. jMetal: A java framework for developing multiobjective optimization metaheuristics. Technical Report ITI2006-10, Departamento de Lenguajes y Ciencias de la Computación, University of Málaga, 2006.

[7] H. Eskandari, C. D. Geiger, and R. Bird. Handling uncertainty in evolutionary multiobjective optimization: SPGA. In Proc. of IEEE Congress on Evol. Comp., 2007.

[8] H. georg Beyer. Evolutionary algorithms in noisy environments: Theoretical issues and guidelines for practice. $\mathrm{El}$ sevier Computer Methods in Applied Mechanics and Engineering, 186(2-4):239-267, 2000.

[9] C. K. Goh and K. C. Tan. Noise handling in evolutionary multi-objective optimization. In Proc. of IEEE Congress on Evol. Comp., 2006.

[10] Y. Jin and J. Branke. Evolutionary optimization in uncertain environments-a survey. IEEE Trans. on Evol. Comp., 9:303$317,2005$.

[11] N. Srinivas and K. Deb. Multiobjective function optimization using nondominated sorting genetic algorithms. MIT Evol. Comp. J., 2(3), 1995. 


\begin{tabular}{|c|c|c|c|c|c|c|}
\hline Problem & $\mathcal{C}($ NSGA-II-A, NSGA-II-U) & $\mathcal{C}($ NSGA-II-U, NSGA-II-A) & $\mathcal{C}($ NSGA-III-U, NSGA-II-N) & $\mathcal{C}(\mathbf{N S G A}-\mathrm{II}-\mathbf{N}, \mathbf{N S G A - I I - U})$ & $\mathcal{C}($ NSGA-II-A, NSGA-II-N) & $\mathcal{C}($ NSGA-II-N, NSGA-II-A $)$ \\
\hline ZDT1-1-N & $3.00 e-03_{1.3 e}-04$ & $0.00 e+00_{1.0 e+00}$ & $0.00 e+00_{0.0 e+00}$ & $0.00 e+00_{1.0 e+00}$ & $0.00 e+00_{0.0 e+00}$ & $0.00 e+00_{0.0 e+00}$ \\
\hline ZDT1-4-N & $2.27 e-021.7 e-03$ & $3.17 \mathrm{e}-021.0 \mathrm{e}+00$ & $9.62 e-039.3 e-04$ & $2.57 \mathrm{e}-021.0 \mathrm{e}+00$ & $7.69 e-031.7 e-03$ & $6.63 \mathrm{e}-021.2 \mathrm{e}-02$ \\
\hline ZDT2-1-N & $0.00 e+000.0 e+00$ & $0.00 e+00_{1.0 e+00}$ & $0.00 e+000.0 e+00$ & $0.00 e+00_{1.0 e+00}$ & $0.00 e+000.0 e+00$ & $0.00 e+00_{0} .0 e+00$ \\
\hline ZDT2-4-N & $6.67 e-041.3 e-05$ & $0.00 e+001.0 e+00$ & $0.00 e+000.0 e+00$ & $0.00 e+001.0 e+00$ & $0.00 e+000.0 e+00$ & $0.00 e+000.0 e+00$ \\
\hline ZDT3-1-N & $4.13 e-022.5 e-03$ & $0.00 e+001.0 e+00$ & $0.00 e+000.0 e+00$ & $0.00 e+001.0 e+00$ & $3.20 e-025.6 e-03$ & $0.00 e+000.0 e+00$ \\
\hline ZDT3-4-N & $1.53 e-025.6 e-04$ & $1.29 e-011.0 e+00$ & $4.71 e-029.9 e-03$ & $3.03 e-021.0 e+00$ & $9.63 e-031.7 e-03$ & $8.30 e-021.4 e-02$ \\
\hline ZDT1-1-U & $3.67 \mathrm{e}-03_{1.2 \mathrm{e}-04}$ & $0.00 e+00_{1.0 e+00}$ & $0.00 e+000.0 e+00$ & $0.00 e+001.0 e+00$ & $2.13 e-031.3 e-04$ & $0.00 e+000.0 e+00$ \\
\hline ZDT1-4-U & $2.33 e-033.1 e-05$ & $0.00 e+001.0 e+00$ & $0.00 e+000.0 e+00$ & $0.00 e+001.0 e+00$ & $8.29 e-03_{4.4 e}-04$ & $0.00 e+00_{0} 0 e+00$ \\
\hline ZDT2-1-U & $0.00 e+000.0 e+00$ & $0.00 e+001.0 e+00$ & $0.00 e+000.0 e+00$ & $0.00 e+001.0 e+00$ & $0.00 e+000.0 e+00$ & $0.00 e+000.0 e+00$ \\
\hline ZDT2-4-U & $0.00 e+000.0 e+00$ & $0.00 e+001.0 e+00$ & $0.00 e+000.0 e+00$ & $0.00 e+001.0 e+00$ & $0.00 e+000.0 e+00$ & $0.00 e+000.0 e+00$ \\
\hline ZDT3-1-U & $5.23 e-022.7 e-03$ & $0.00 e+001.0 e+00$ & $0.00 e+000.0 e+00$ & $0.00 e+001.0 e+00$ & $026.5 e-03$ & $0.00 e+000.0 e+00$ \\
\hline ZDT3-4-U & $5.70 e-025.7 e-03$ & $0.00 e+001.0 e+00$ & $0.00 e+000.0 e+00$ & $0.00 e+001.0 e+00$ & $6.08 e-021.0 e-02$ & $0.00 e+000.0 e+00$ \\
\hline ZDT1-1-P & $3.33 e-038.2 e-05$ & $0.00 e+001.0 e+00$ & $0.00 e+000.0 e+00$ & $0.00 e+001.0 e+00$ & $5.81 e-03_{4} .7 e-04$ & $0.00 e+000.0 e+00$ \\
\hline ZDT1-4-P & $4.67 e-034.1 e-04$ & $0.00 e+001.0 e+00$ & $0.00 e+000.0 e+00$ & $0.00 e+001.0 e+00$ & $0.00 e+000.0 e+00$ & $0.00 e+000.0 e+00$ \\
\hline ZDT2-1-P & $0.00 e+000.0 e+00$ & $0.00 e+001.0 e+00$ & $0.00 e+000.0 e+00$ & $0.00 e+001.0 e+00$ & $0.00 e+000.0 e+00$ & $0.00 e+000.0 e+00$ \\
\hline ZDT2-4-P & $0.00 e+00_{0.0 e+00}$ & $0.00 e+001.0 e+00$ & $0.00 e+000.0 e+00$ & $0.00 e+001.0 e+00$ & $0.00 e+00_{0} .0 e+$ & $0.00 e+00_{0} .0 e+00$ \\
\hline ZDT3-1-P & $3.30 e-022.6 e-03$ & $0.00 e+001.0 e+00$ & $0.00 e+000.0 e+00$ & $3.33 \mathrm{e}-041.0 \mathrm{e}+00$ & $3.85 e-026.9$ & $0.00 e+00_{0.0 e+00}$ \\
\hline ZDT3-4-P & $5.20 e-023.0 e-03$ & $0.00 e+001.0 e+00$ & $0.00 e+00_{0.0 e+00}$ & $0.00 e+00_{1.0 e+00}$ & $6.94 \mathrm{e}-021.4 \mathrm{e}-02$ & $0.00 e+000.0 e+00$ \\
\hline
\end{tabular}

Table 11: $\mathcal{C}$-metric on Bi-objectives Problems

\begin{tabular}{|c|c|c|c|c|c|c|}
\hline Problem & $\mathcal{C}($ NSGA-II-A, NSGA-II-U) & $\mathcal{C}$ (NSGA-II-U, NSGA-II-A) & $\mathcal{C}$ (NSGA-II-U, NSGA-II-N) & $\mathcal{C}$ (NSGA-II-N, NSGA-II-U) & $\mathcal{C}$ (NSGA-II-A, NSGA-II-N) & $\mathcal{C}$ (NSGA-II-N, NSGA-II-A) \\
\hline DTLZ1-1-N & $5.60 \mathrm{e}-023.2 \mathrm{e}-03$ & $0.00 e+001.0 e+00$ & $9.33 e-038.9 e-04$ & $1.00 e-031.0 e+00$ & $6.60 e-023.0 e-03$ & $0.0 e+00$ \\
\hline DTLZ1-4-N & $6.67 \mathrm{e}-023.2 \mathrm{e}-03$ & $0.00 e+001.0 e+00$ & $4.67 e-03_{2.8 e}-04$ & $2.67 e-031.0 e+00$ & $6.40 e-022.7 e-03$ & $0.00 e+000.0 e+00$ \\
\hline DTLZ3-1-N & $2.42 \mathrm{e}-01_{1.0 e-02}$ & $0.00 e+001.0 e+00$ & $1.00 e-03_{9.0 e-06}$ & $3.67 \mathrm{e}-03_{1.0 e}+00$ & $2.68 \mathrm{e}-01_{1.4 \mathrm{e}-02}$ & $0.00 e+000.0 e+00$ \\
\hline DTLZ3-4-N & $1.93 e-016.1 e-03$ & $0.00 e+00_{1.0 e+00}$ & $3.33 e-039.6 e-05$ & $0.00 e+001.0 e+00$ & $2.08 \mathrm{e}-018.1 \mathrm{e}-03$ & $0.00 e+000.0 e+00$ \\
\hline DTLZ7-1-N & $0.00 e+00_{0.0 e+00}$ & $0.00 e+001.0 e+00$ & $0.00 e+000.0 e+00$ & $0.00 e+001.0 e+00$ & $0.00 e+000.0 e+00$ & $0.00 e+000.0 e+00$ \\
\hline DTLZ7-4-N & $0.00 e+000.0 e+00$ & $0.00 e+001.0 e+00$ & $0.00 e+000.0 e+00$ & $0.00 e+001.0 e+00$ & $0.00 e+000.0 e+00$ & $0.00 e+000.0 e+00$ \\
\hline DTLZ1-1-U & $7.57 e-023.0 e-03$ & $0.00 e+001.0 e+00$ & $5.33 e-032.5 e-04$ & $3.00 e-031.0 e+00$ & $6.83 e-023.8 e-03$ & $0.00 e+000.0 e+00$ \\
\hline DTLZ1-4-U & $4.80 e-021.7 e-03$ & $0.00 e+001.0 e+00$ & $3.67 e-03_{2} .2 e-04$ & $5.33 e-031.0 e+00$ & $4.73 e-022.0 e-03$ & $0.00 e+000.0 e+00$ \\
\hline DTLZ3-1-U & $2.56 e-011.2 e-02$ & $0.00 e+00_{1.0 e+00}$ & $3.00 e-038.8 e-05$ & $2.33 e-03_{1} .0 e+00$ & $2.44 e-019.4 e-03$ & $0.00 e+000.0 e+00$ \\
\hline DTLZ3-4-U & $2.34 \mathrm{e}-01_{5} .4 \mathrm{e}-03$ & $0.00 e+001.0 e+00$ & $1.00 e-039.0 e-06$ & $6.67 e-041.0 e+00$ & $2.35 e-019.5 e-03$ & $0.00 e+000.0 e+00$ \\
\hline DTLZ7-1-U & $0.00 e+00_{0.0 e+00}$ & $0.00 e+001.0 e+00$ & $0.00 e+000.0 e+00$ & $0.00 e+001.0 e+00$ & $0.00 e+00_{0} .0 e+00$ & $0.00 e+000.0 e+00$ \\
\hline DTLZ7-4-U & $0.00 e+000.0 e+00$ & $0.00 e+00_{1.0 e+00}$ & $0.00 e+000.0 e+00$ & $0.00 e+001.0 e+00$ & $0.00 e+000.0 e+00$ & $0.00 e+000.0 e+00$ \\
\hline DTLZ1-1-P & $7.63 e-023.4 e-03$ & $0.00 e+001.0 e+00$ & $3.00 e-038.8 e-05$ & $2.00 e-031.0 e+00$ & $7.70 e-024.2 e-03$ & $0.00 e+000.0 e+00$ \\
\hline DTLZ1-4-P & $4.07 e-021.8 \mathrm{e}-03$ & $0.00 e+001.0 e+00$ & $4.33 e-031.6 e-04$ & $3.00 e-031.0 e+00$ & $5.67 e-023.1 e-03$ & $0.00 e+000.0 e+00$ \\
\hline DTLZ3-1-P & $2.22 \mathrm{e}-011.1 \mathrm{e}-02$ & $0.00 e+001.0 e+00$ & $2.33 e-035.8 e-05$ & $6.67 e-031.0 e+00$ & $2.15 e-016.9 e-03$ & $0.00 e+000.0 e+00$ \\
\hline DTLZ3-4-P & $2.22 \mathrm{e}-011.1 \mathrm{e}-02$ & $0.00 e+001.0 e+00$ & $3.67 e-03_{1.3 e-04}$ & $3.33 e-03_{1} .0 e+00$ & $2.25 e-018.8 e-03$ & $0.00 e+000.0 e+00$ \\
\hline DTLZ7-1-P & $0.00 e+000.0 e+00$ & $0.00 e+001.0 e+00$ & $0.00 e+000.0 e+00$ & $0.00 e+001.0 e+00$ & $0.00 e+000.0 e+00$ & $0.00 e+000.0 e+00$ \\
\hline DTLZ7-4-P & $0.00 e+000.0 e+00$ & $0.00 e+001.0 e+00$ & $0.00 e+000.0 e+00$ & $0.00 e+001.0 e+00$ & $0.00 e+000.0 e+00$ & $0.00 e+00_{0.0 e+00}$ \\
\hline
\end{tabular}

Table 12: $\mathcal{C}$-metric on Tri-objectives Problems

[12] J. Teich. Pareto-front exploration with uncertain objectives. In Proc. of Int'l Conf. on Evol. Multi-Criterion Optimization, 2001.

[13] J. R. K. Urmila M. Diwekar. Efficient sampling technique for optimization under uncertainty. Wiley AIChE Journal, 43(2), 1997.

[14] D. V. Veldhuizen and G. Lamont. Multiobjective evolutionary algorithm research: A history and analysis. Technical Report TR-98-03, Dept. Elec. Comput. Eng., Air Force Inst. Technol., 1998.

[15] M. Wormington, C. Panaccione, K. M. Matney, and D. K. Bowen. Characterization of structures from $\mathrm{x}$-ray scattering data using genetic algorithms. JSTOR Philosophical Transactions, 357(1761):2827-2848, 1999.

[16] E. Zitzler, K. Deb, and L. Thiele. Comparison of multiobjective evolutionary algorithms: Empirical results. IEEE Trans. on Evol. Comp., 8:173-195, 2000.

[17] E. Zitzler and L. Thiele. Multiobjective evolutionary algorithms: a comparative case study and the strength pareto approach. IEEE Trans. Evol. Comp., 3(4):257-271, 1999.

[18] E. Zitzler, L. Thiele, M. Laummanns, C. Fonseca, and G. da Fonseca. Performance assesment of multiobjective optimizars: An analysis and review. IEEE Trans. on Evol. Сотр., 3:257-271, 1999. 